12

\title{
Особенности нелинейной диффузии сильного импульсного магнитного поля вблизи края проводника
}

\author{
() С.И. Кривошеев, С.Г. Магазинов, Г.А. Шнеерсон \\ Санкт-Петербургский политехнический университет Петра Великого, Санкт-Петербург, Россия \\ E-mail: ksi.mgd@mail.ru
}

Поступило в Редакцию 28 августа 2018 г.

Рассмотрена нелинейная диффузия сильного магнитного поля в проводник, границы которого образуют прямоугольный двугранный угол. В поперечном поле с индукцией, перпендикулярной ребру угла, максимум плотности тока имеет место не вблизи ребра, как при линейной диффузии, а в глубине проводника. В продольном поле с индукцией, параллельной ребру, плотность тока в вершине угла при диффузии сильного поля равна нулю, как и в слабом поле, но местоположение максимумов плотности тока, а также температуры и силы Лоренца при нарастании поля смещаются в толщу проводника.

DOI: 10.21883/PJTF.2019.03.47272.17504

Вблизи кромок проводников прямоугольного сечения и в местах соединения токоведущих шин с одновитковыми соленоидами имеет место двумерная диффузия импульсного магнитного поля. Линейный режим такой диффузии в определенной мере описан в литератуpe [1-3], но нелинейная двумерная диффузия не исследована, хотя влияние этого процесса на нагрев и деформацию проводников в сильном магнитном поле может быть весьма существенным. Эти эффекты приводят к особенностям деформации и разрушения проводников в сильных магнитных полях, создаваемых в устройствах для технологических приложений и физических исследований $[4,5]$.

Диффузия поля рассмотрена далее на примере проводника, границы которого образуют прямоугольный двугранный угол. Характер распределения тока вблизи ребра двугранного угла определяется ориентацией вектора индукции внешнего поля. Целесообразно рассмотреть конфигурации с поперечным и продольным полем. В первой указанный вектор направлен перпендикулярно, а во второй - параллельно ребру (рис. 1, $a$ и 2,a).

Можно отметить различие характера диффузии в этих двух случаях, имеющее место в линейном режиме. В поперечном магнитном поле, когда ток направлен параллельно ребру, его плотность принимает максимальное значение на кромке [6]. В продольном поле плотность тока равна нулю в вершине двугранного угла. В этом случае вдоль биссектрисы угла имеет место немонотонное распределение плотности тока. В частности, при включении поля толчком расстояние $r_{M}$ от вершины прямого угла до местоположения максимума характеризуется зависимостью $r_{M}(t)=1.75\left(\rho_{0} t / \mu_{0}\right)^{1 / 2}$, где $\rho_{0}$ - удельное сопротивление проводника, $t$ время от начала процесса, $\mu_{0}=4 \pi \cdot 10^{-7} \mathrm{H} / \mathrm{m}[1]$.

В настоящей работе рассматривается влияние роста удельного сопротивления, вызванного нагревом проводника током, на характер двумерной диффузии. Одномерная нелинейная диффузия, обусловленная этим эффектом, описана в ряде работ [1]. Отмечено, что поле прони- кает в среду на расстояние порядка $\Delta=B / B_{0}\left(\rho_{0} t / \mu_{0}\right)^{1 / 2}$. Здесь $B_{0}$ - характерная индукция, определяемая выражением $B_{0}=\left(2 \mu_{0} / \beta\right)^{1 / 2}$, где $\beta-$ коэффициент, фигурирующий в формуле, которая обычно используется для аппроксимации зависимости удельного сопротивления от объемной плотности энергии джоулева нагрева $\Delta q^{\prime}$

$$
\rho=\rho_{0}\left(1+\beta \Delta q^{\prime}\right) .
$$

Для меди $B_{0} \approx 42$ Т. Особенностью одномерной нелинейной диффузии является немонотонная зависимость плотности тока от расстояния до границы: вследствие нагрева среды ток вопреки скин-эффекту вытесняется в глубь среды, где имеет место максимум плотности тока. Объемная плотность тепловой энергии достигается на границе. При монотонно нарастающем поле она принимает значение $\Delta q^{\prime}=\eta B_{e}^{2} /\left(2 \mu_{0}\right)$, где коэффициент $\eta$ близок к единице [1]. Представляет интерес, насколько в случае нелинейной двумерной диффузии изменятся распределение тепловой энергии в скин-слое и ее значение вблизи вершины угла. При этом есть основания предполагать, что нагрев среды будет по-разному проявляться в случаях продольного и поперечного полей.

Особенности двумерной диффузии исследованы путем компьютерного моделирования. Влияние движения среды в процессе деформации проводника при расчетах не учитывалось. Основанием для этого являются оценки числа Рейнольдса. Они показывают, что в скин-слое, где может иметь место течение металла вдоль границы, это число много меньше единицы. В системе координат, связанных с границей проводника, магнитное поле описывается уравнениями Максвелла для неподвижной среды. Исследовано поле проводника, поперечное сечение которого имеет форму квадрата со стороной $a=5 \mathrm{~mm}$.

Решение уравнений Максвелла выполнено на расчетной сетке, состоящей из равномерно распределенных квадратных элементов размером $0.05 \mathrm{~mm}$. В рассмотренных режимах глубина проникновения магнитного поля (толщина скин-слоя) существенно больше размеров элементов сетки. Объемная плотность мощности джоулева 

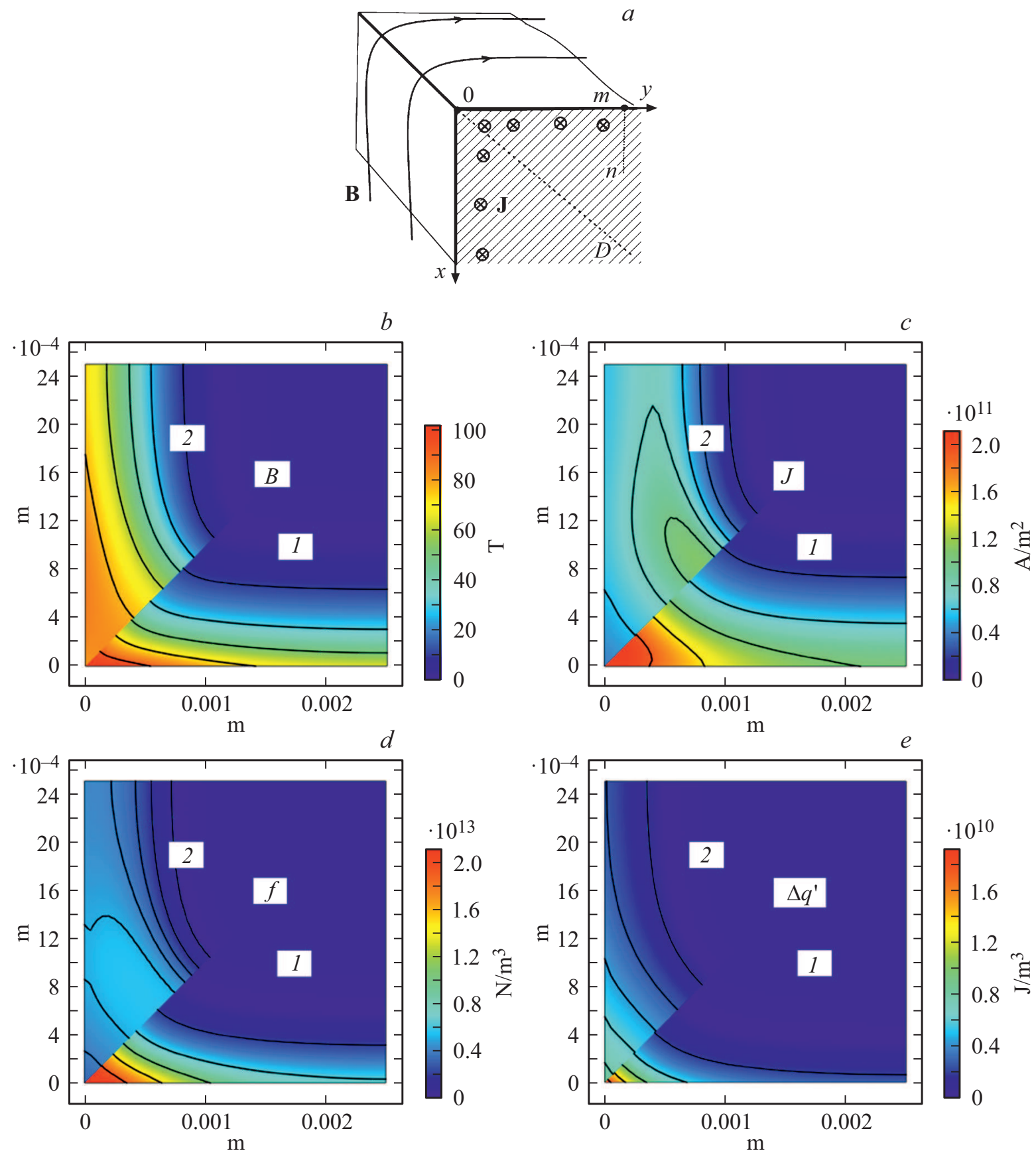

Pис. 1. Диффузия поперечного к углу проводника магнитного поля: ориентация магнитного поля $\mathbf{B}$ и плотности тока $\mathbf{J}(a)$, распределение индукции $|\mathbf{B}|(b)$, плотности тока $|\mathbf{J}|(c)$, силы Лоренца $|\mathbf{f}|(d)$, плотности энергии тепловыделения $\Delta q^{\prime}$ в момент времени, соответствующий максимуму тока $(e) .1$ - для постоянного, 2 - для рассчитываемого по формуле $(1)$ удельного сопротивления. Приведены линии равных значений соответствующих параметров.

нагрева рассчитывалась по формуле $d\left(\Delta q^{\prime}\right) / d t=\rho J^{2}$, где $\Delta q^{\prime}$ - приращение объемной плотности тепловой энергии, $J-$ плотность тока. При расчете учитывалась зависимость удельного сопротивления от объемной плотности тепловой энергии (1). Рассчитанное значение этой величины позволяет найти приращение температуры: $\Delta T=\Delta q^{\prime} /\left(\gamma C_{p}(T)\right)$, где $\gamma$ и $C_{p}(T)$ - плотность и теплоемкость соответственно. Теплоемкость в диапазоне температур 25-1300 K задавалась выражением

$$
\begin{aligned}
C_{p}(T)= & \alpha_{0}+\alpha_{1}\left(T-T_{\min }\right) / 100+\alpha_{2}\left(\left(T-T_{\min }\right) / 100\right)^{2} \\
& +\alpha_{3}\left(\left(T-T_{\min }\right) / 100\right)^{3},
\end{aligned}
$$

где коэффициенты $\alpha_{i}$ взяты из работы [7], а при температурах выше $1300 \mathrm{~K} C_{p}$ принята постоянной, соответствующей температуре $1300 \mathrm{~K}$. 

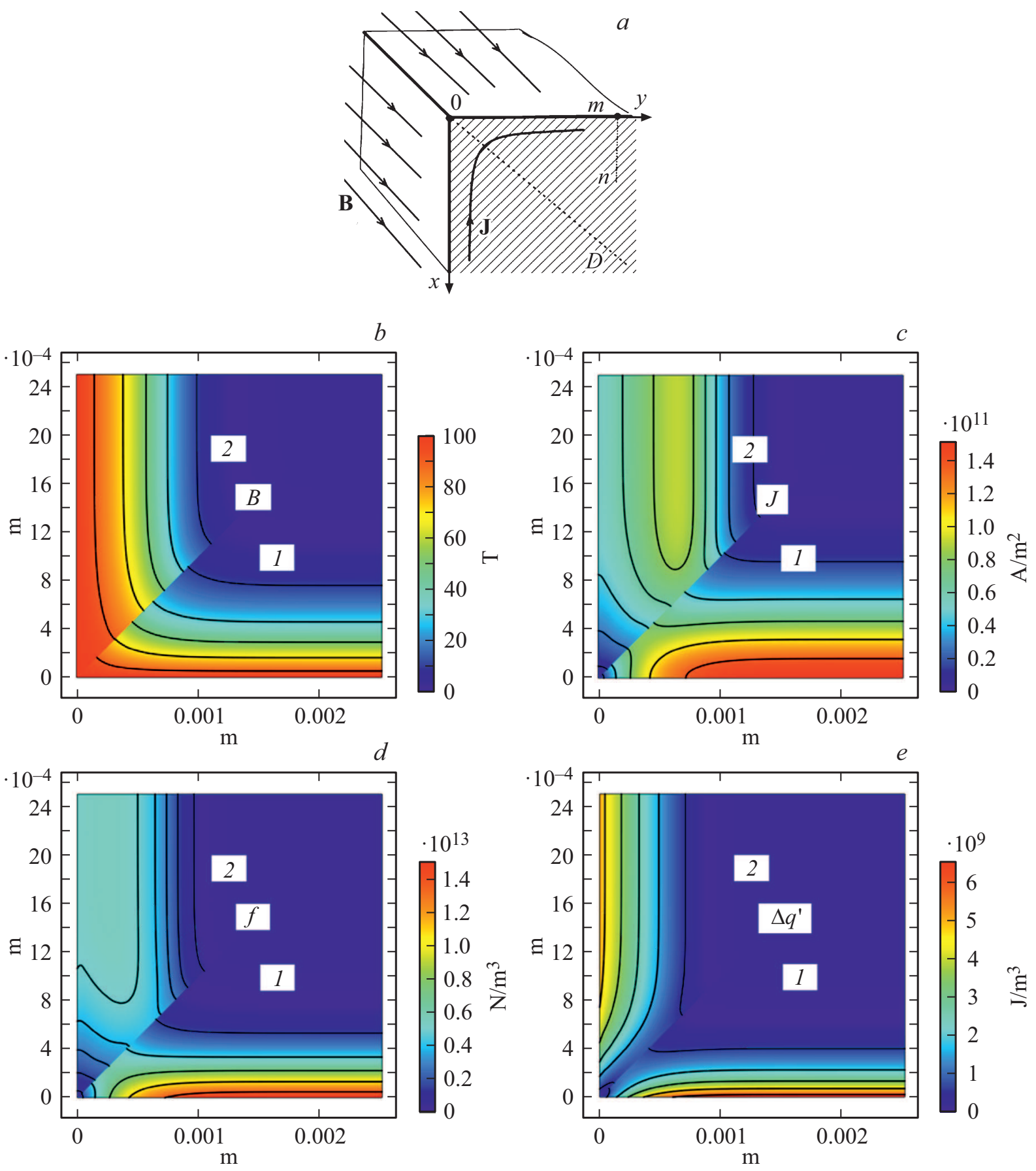

Pис. 2. То же, что на рис. 1, в случае диффузии продольного (вдоль угла проводника) магнитного поля.

Рассмотрим диффузию поперечного поля.

В случае поперечного поля принято, что проводник расположен в центре круга с радиусом $R$, на границе которого задано условие магнитной изоляции $B_{r}=0$. Это условие приемлемо в случае, когда $R \gg a$ и внешнее поле мало отличается от поля проводника круглого сечения. Симметрия магнитной системы позволила ограничиться решением уравнений Максвелла для четверти круга с включением в нее четверти квадратного сечения проводника. При диффузии поперечного поля на гра- нице проводника выполнялось условие непрерывности нормальной и касательной компонент индукции. На границах симметрии расчетной области задано условие отсутствия касательной компоненты индукции, являющееся следствием симметрии системы. В качестве внешнего воздействия задан полный синусоидальный ток с амплитудой $300 \mathrm{kA}$ (в области, соответствующей четверти квадрата) и периодом $50 \mu \mathrm{s}$.

На рис. 1 представлены пространственные распределения абсолютных значений индукции $B$, плотности тока $J$, 

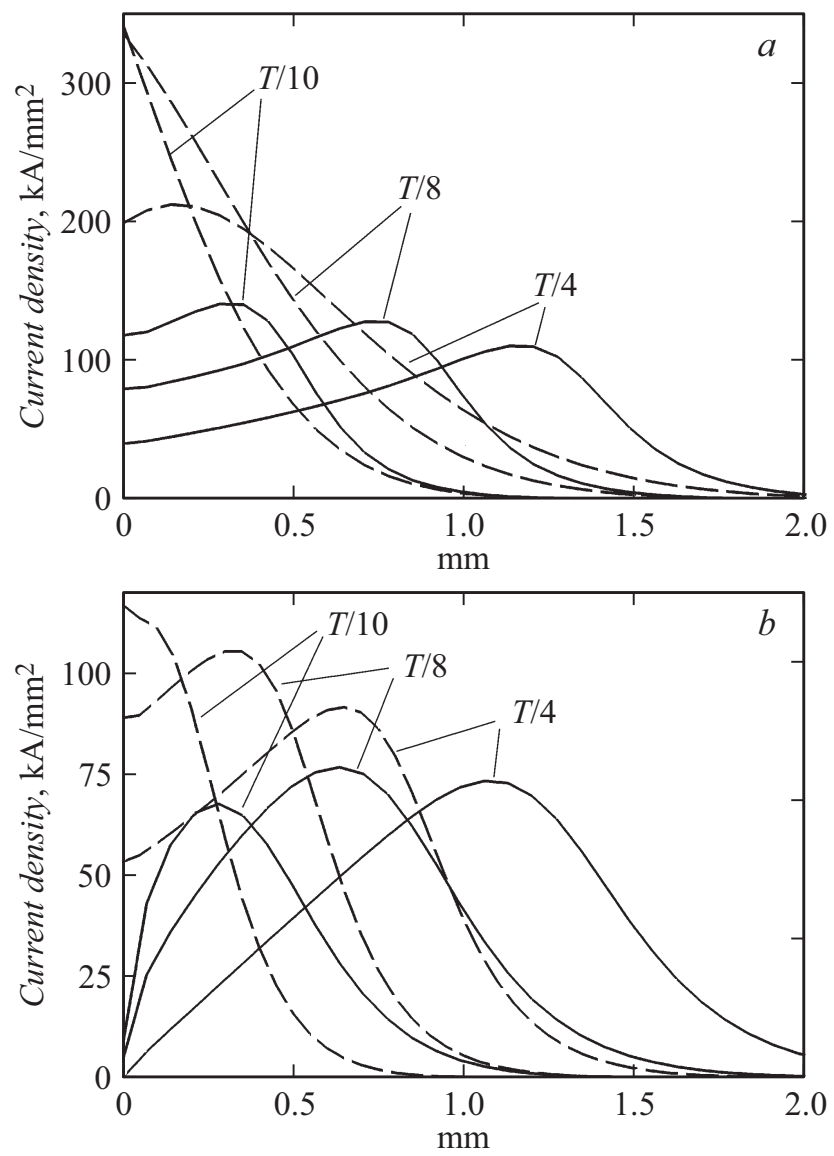

Рис. 3. Распределение плотности тока $|\mathbf{J}|$ в моменты времени $T / 10, T / 8, T / 4 . a-$ вдоль биссектрисы угла (линия $0 D$ ) при диффузии поперечного поля. Штриховые линии построены для постоянной проводимости, сплошные линии - с учетом влияния нагрева на удельное сопротивление (см. формулу (1)); $b$ - при диффузии продольного поля с учетом влияния нагрева на удельное сопротивление. Штриховые линии - вдоль линии $m n$ (одномерный случай), сплошные - по биссектрисе угла вдоль линии $0 D$.

силы Лоренца $f=J B$, а также плотности тепловой энергии $\Delta q^{\prime}$. В областях 1 показано распределение соответствующих величин при линейной диффузии, рассчитанное при постоянном сопротивлении, а в областях 2 - распределение при нелинейной диффузии, рассчитанное с учетом температурной зависимости сопротивления. Зависимости построены для момента времени, соответствующего максимуму тока с амплитудой $300 \mathrm{kA}$. К этому моменту индукция поля в точках $m$ и 0 достигает значения $80 \mathrm{~T}$, существенно превышающего характерную индукцию $B_{0}$. В таком поле проявляются особенности нелинейной двумерной диффузии.

Как было отмечено выше, при нелинейной диффузии снижение проводимости металла в области, прилегающей к границе, приводит к вытеснению тока в толщу проводника, где формируется максимум плотности тока. Это подтверждают зависимости распределения плотности тока вдоль биссектрисы угла $0 D$ при на- растающем поле, построенные для разных моментов времени (рис. 3,a). Рост сопротивления при нагреве приводит к сглаживанию неоднородности распределения плотности тока по границе, свойственного двумерной диффузии: отношение плотностей тока в точках 0 и $m$ $\delta(0) / \delta(m)$ при линейной диффузии составляет 1.90, а при нелинейной -0.69 .

Пространственное распределение других величин также показывает существенное отличие линейного режима от нелинейного. В частности, отношения приращений объемной плотности тепловой энергии в указанных точках в момент времени, соответствующий максимуму тока, составляют 7.21 и 3.19. При этом приращение температуры в точке 0 в линейном режиме составляет $4261 \mathrm{~K}$, а в нелинейном - $2202 \mathrm{~K}$. Менее контрастным становится и распределение объемной силы. При заданной форме импульса тока и размерах проводника отношение ее значений в вершине угла и в точке $m$ в момент времени, соответствующий максимуму тока, в линейном режиме составляет 2.89. В нелинейном режиме эта неоднородность сглаживается в большей степени, чем неоднородность распределения плотности тока: указанное отношение становится равным 0.74 .

Рассмотрим диффузию продольного поля.

В случае продольного поля решение отличалось выбором граничных условий. На границах проводника $0 x$ и $0 y$ (рис. 2,a) задано синусоидальное магнитное поле $B_{z}$ c амплитудой индукции $100 \mathrm{~T}$, нарастающее начиная с нуля по синусоидальному закону с периодом колебаний $T=50 \mu \mathrm{s}$. На границах симметрии расчетной области задано условие $\mathbf{n} \cdot \mathbf{A}=0$, где $\mathbf{A}-$ векторный потенциал магнитного поля, $\mathbf{n}$ - вектор нормали.

Результаты расчета представлены на рис. 2 и рис. $3, b$. Ранее было отмечено, что при линейной диффузии продольного поля плотность тока в точке 0 на ребре проводника равна нулю. Этот эффект сохраняется и при нелинейной диффузии. Об этом свидетельствуют пространственные распределения тока, объемной плотности тепловой энергии и силы Лоренца, показанные на рис. 2. Роль указанного эффекта проявляется в резком различии распределения плотности тока по нормали $m n$ и по биссектрисе угла. Распределения плотности тока на рис. $3, b$ построены для разных моментов времени для поля с индукцией $100 \mathrm{~T}$, когда диффузия является нелинейной. Распределение плотности тока по нормали близко к рассчитанному для одномерной диффузии. Характерным различием распределений плотности тока по указанным направлениям является то, что как отмечалось, в вершине угла плотность тока принимает нулевое значение в линейном и нелинейном случае. Это приводит к существенно разному распределению энерговыделения в зонах, занятых током. При диффузии по нормали (вдали от угла — в точке $n$ ) в момент времени, соответствующий максимуму тока, достигается температура $1471 \mathrm{~K}$, близкая к точке плавления меди, тогда как вдоль линии $0 D$ существенного роста температуры не наблюдается: в точке 0 температура остается 
равной начальной. Дополнительные расчеты с учетом теплопроводности показали, что в рассмотренном примере температура в точке 0 возрастает лишь на $73 \mathrm{~K}$.

Общей чертой двумерной нелинейной диффузии является бо́льшая скорость перемещения максимума тока в направлении биссектрисы угла, чем по нормали к границе. В рассчитанных режимах диффузии продольного и поперечного поля скорость смещения максимума плотности тока возрастала приблизительно в 1.5 раза.

Резкое снижение плотности тока, энерговыделения и электромагнитной силы в угловой точке является принципиальным отличием двумерной диффузии продольного поля от диффузии поперечного. Края токоведущих шин генераторов больших импульсных токов ориентированы параллельно линиям тока и подвергаются воздействию поперечного поля. Они испытывают интенсивные локальные тепловые и механические нагрузки в процессе диффузии этого поля. Напротив, в одновитковых магнитах в месте соединения магнита с подводящими шинами индукция параллельна ребру угла. Даже в очень сильном поле участок вблизи ребра, находящегося в продольном поле, подвергается менее интенсивному тепловому и механическому воздействию. Он сохраняет свою конфигурацию и мало смещается даже при наличии интенсивного пластического течения остальной части проводника, деформированного в поле с индукцией 117 T [8]. Конфигурация окрестностей ребра сохраняется в экспериментах с более сильными полями [9] и даже в том случае, когда происходит электрический взрыв магнита. Это подтверждается рентгенограммами процесса разрушения одновиткового соленоида, полученными при генерации магнитного поля с амплитудой индукции $350 \mathrm{~T}[10]$.

Таким образом, рассмотренный эффект носит достаточно общий характер и проявляется в широком классе электрофизических устройств, работающих в сильном импульсном магнитном поле. Этот эффект должен учитываться при разработке устройств для получения импульсных магнитных полей и может иметь особое значение при создании устройств для передачи больших импульсных токов к нагрузке малых размеров [11].

Моделирование процессов диффузии проведено в программной среде Comsol Multiphysics 5.3 с использованием ресурсов суперкомпьютерного центра „Политехнический“: RSC Tornado (параметры одного узла $2 \times \mathrm{CPU}$ with 14 cores (2xXeonE5-2697v3 2.6 GHz 64 GBRAM)).

Работа выполнена при поддержке гранта РНФ № 18-19-00230.

\section{Список литературы}

[1] Shneerson G.A., Dolotenko M.I., Krivosheev S.I. Strong and superstrong pulsed magnetic fields generation. De Gruyter studies in mathematical physics. Berlin-Boston: de Gruyter, 2014. $429 \mathrm{p}$.

[2] Chaikovsky S.A., Oreshkin V.I., Datsko I.M., Labetskaya N.A., Rybka D.V., Ratakhin N.A. // Phys. Plasmas. 2015. V. 22. N 11. P. 112704.
[3] Гаранин С.Ф., Иванова Г.Г., Кармишин Д.В., Софронов В.Н. // Приклад. механика и техн. физика. 2005. Т. 46. № 2. C. 5-12.

[4] Krivosheev S.I., Magazinov S.G., Alekseev D.I. // IEEE Trans. Plasma Sci. 2018. V. 46. N 4. P. 1054-1057.

[5] Selemir V.D., Tatsenko O.M., Platonov V.V., Filippov A.V., Bychkova E.A., Krivosheev S.I., Shneerson G.A. // IEEE Trans. Plasma Sci. 2015. V. 43. N 2. P. 688-691.

[6] Shneerson G.A., Belozerov I.A. Edge effect for transverse electromagnetic field penetration into a conductor // 14th Int. Conf. on megagauss magnetic field generation and related topics (MEGAGAUSS 2012). IEEE, 2012. DOI: 10.1109/MEGAGAUSS.2012.6781414

[7] White G.K., Collocott S.J. // J. Phys. Chem. Data. 1984. V. 13. N 4. P. $1251-1257$.

[8] Шнеерсон Г.А. // ЖТФ. 1962. Т. 32. В. 9. С. 1153-1156.

[9] Бочаров Ю.Н., Кривочеев С.И., Кручинин А.И. Титков В.В., Шнеерсон Г.А. Получение сверхсильных импульсных магнитных полей в одновитковых тонкостенных соленоидах. // Третья Международная конференция по генерации мегагауссных магнитных полей и родственным экспериментам. / Под ред. В.М. Титова, Г.А. Швецова. М.: Наука, 1983. С. 77-83.

[10] Кривошеев С.И., Титков В.В., Шнеерсон Г.А. // ЖТФ. 1997. T. 67. B. 4. P. 32-47.

[11] Rose D.V., Welch D.R., Madrid E.A., Miller C.L., Clark R.E., Stygar W.A., Struve K., Corcoran P.A., Whitney B. Electromagnetic wave propagation through the ZR Z-pinch accelerator // 7th Int. Conf. on dense Z-pinches. AIP Conf. Proc. 2009. V. 1088. N 1. P. 263-266. 